\title{
ON GENERALIZED APPROXIMATE CONVEX FUNCTIONS AND VARIATIONAL INEQUALITIES
}

\author{
BhuWan Chandra Joshi*
}

\begin{abstract}
In this paper, we consider a vector optimization problem involving locally Lipschitz generalized approximately convex functions and provide several concepts of approximate efficient solutions. We formulate approximate vector variational inequalities of Minty and Stampacchia type under the framework of Clarke subdifferentials and use these inequalities as a tool to characterize an approximate efficient solution of the vector optimization problem.
\end{abstract}

Mathematics Subject Classification. 49J30, 47H09, 47H10, 47J20.

Received February 17, 2020. Accepted December 9, 2020.

\section{INTRODUCTION}

The variational inequality problem includes many interesting classes of variational problems with applications in game theory, economics and engineering. The basic prototype is smooth convex optimization. Other problems which can be formulated as variational inequalities, include systems of equations, complementarity problems, saddle-point problems and many equilibrium problems. We refer the reader to Chapter 1 of [7] and [8] for an extensive review of applications of the variational inequality problem in economics and engineering. For recent developments in variational inequalities, we refer to $[4,9,14,27]$ and references therein.

The approximation procedures are of immense importance in optimization theory, because sometimes it is practically impossible or computationally very expensive to find out an exact solution. Thus, approximate efficient solutions help to overcome the difficulties arise due to the computational inaccuracies and the modeling limitations. In 1984, Loridan [16] introduced the concept of epsilon efficient solutions for the vector optimization problems. Later, White [29] proposed several concepts of approximate solutions for the vector optimization problems through scalarization. Recently, a concept of approximate efficient solution has been introduced by Mishra and Laha [18] and characterized using approximate vector variational inequalities of Stampacchia and Minty type under assumptions of approximately straight functions. Later, Wang et al. [28] proved that the solutions of generalized vector variational-like inequalities in terms of the generalized Jacobian are the generalized quasi-efficient solutions of nonsmooth multi-objective programming problems under the higher-order generalized invexity assumptions.

In the last three decades, several definitions extending the concept of convexity of a function have been introduced by many researchers. Usually, generalized convex functions have been introduced in order to weaken

Keywords. Clarke subdifferentials, vector optimization, approximate vector variational inequalities.

Department of Mathematics, Graphic Era (Deemed to be University), Dehradun 248002, India.

*Corresponding author: bhuwanjoshi007@gmail.com 
the convexity requirements as much as possible to obtain results related to optimization theory. One of the significant generalization of convex function is invex function (invarient under convex transformations) $[5,12]$. The class of invex functions preserves many properties of the class of convex functions and has shown to be very useful in a variety of applications [17]. Osuna-Gómez et al. [25] introduced the notions of generalized invexity for differentiable functions in a finite-dimensional contex. This generalized invexity has been extended to locally Lipschitz functions using the generalized Jacobian (see [11]). Ivanov [13] provided the concept of second-order invex functions and dealt with the optimal solution of nonlinear programming problem. For more literature on generalized convexity and their applications, we refer to $[1,3,10,19,22,23,26]$ and references therein.

Recently, much attention has been given to the class of approximately convex functions as it helps to relax the convexity or smoothness assumptions for practical applications. In 2000, Ngai et al. [24] introduced the concept of approximate convexity as an extension of the concept of epsilon convexity given by Jofre et al. [15]. Later, Daniilidis and Georgiev [6] showed that a locally Lipschitz function is approximately convex if and only if its Clarke subdifferential is a submonotone operator. Motivated by the earlier work on approximate convex function, Wang et al. [28] introduced several extended approximately invex vector-valued functions of higher order involving a generalized Jacobian. Inspired and motivated by this ongoing research work, we present the concept of approximately invex function. The outline of this paper is as follows: in Section 2, we give some basic definitions and results which will be used in the sequel. In Section 3, we give a variant of the concept of approximate efficient solutions for a vector optimization problem involving locally Lipschitz approximately invex functions and characterize these approximate efficient solutions using approximate vector variational inequalities of Minty and Stampacchia type in terms of the Clarke subdiffferentials.

\section{Preliminaries}

In this section, we recall some known definitions and results which will be used in the sequel. Let $\mathbb{R}^{n}$ be the $n$-dimensional Euclidean space. For any $u:=\left(u_{1}, \ldots, u_{n}\right)$ and $v:=\left(v_{1}, \ldots, v_{n}\right)$, we define:

$$
\begin{aligned}
& u \leqslant v \Longleftrightarrow u-v \in-\mathbb{R}_{+}^{n} \backslash\{0\} ; \\
& u \nless v \Longleftrightarrow u-v \notin-\mathbb{R}_{+}^{n} \backslash\{0\} ; \\
& u<y \Longleftrightarrow u-v \in \operatorname{int} \mathbb{R}_{+}^{n} ; \\
& u \nless v \Longleftrightarrow u-v \notin \operatorname{int} \mathbb{R}_{+}^{n} .
\end{aligned}
$$

Let $B$ be a real Banach space endowed with a norm $\|\bullet\|$ and $B^{*}$ its dual space with a norm $\|\bullet\|^{*}$. We denote by $\langle.,\rangle,.[u, v]$ and $(u, v)$, the dual pair between $B$ and $B^{*}$, the line segment for $u, v \in B$ and the interior of $[u, v]$, respectively. Let $\mathbb{B}(u ; \delta)$ denotes the open ball centered at $u \in B$ with radius $\delta>0$. Let $h: B \rightarrow \mathbb{R}_{\infty}:=\mathbb{R} \cup\{+\infty\}$ be an extended real valued function with domain domh $:=\{u \in B: g(u) \neq+\infty\}$.

Let $f:=\left(f_{1}, \ldots, f_{m}\right): B \rightarrow \mathbb{R}^{m}$ be given. For each $y \in \mathbb{R}^{m}$ the composite function, $(y f): B \rightarrow \mathbb{R}$, is defined by

$$
(y f)(u)=\langle y, f(u)\rangle=\sum_{i=1}^{m} y_{i} f_{i}(u) .
$$

The Clarke directional derivative of $y f$ at $\bar{u}$ in the direction $x \in B$ is given by

$$
(y f)^{o}(\bar{u} ; x)=\lim _{u \rightarrow \bar{u}} \sup _{t \downarrow 0} \frac{\langle y, f(u+t x)-f(u)\rangle}{t} .
$$

The Clarke subdifferential of $f$ at $\bar{u}$ is given by

$$
\partial f(\bar{u})=\left\{\bar{u}^{*} \in L\left(B, \mathbb{R}^{m}\right):(y f)^{o}(\bar{u} ; x) \geqslant\left\langle\bar{u}^{*}(x), y\right\rangle, \forall x \in B, \forall y \in \mathbb{R}^{m}\right\} .
$$

Definition 2.1 ([21]). The subset $X \subseteq \mathbb{R}^{n}$ is said to be invex with respect to $\eta: X \times X \rightarrow \mathbb{R}^{n}$, if for every $u, v \in X, \lambda \in[0,1]$, we have $v+\lambda \eta(u, v) \in X$. 
Definition $2.2([20])$. A locally Lipschitz vector function $f: X \subseteq \mathbb{R}^{n} \rightarrow \mathbb{R}^{m}$ is said to be approximate convex at $\bar{u} \in X$, iff for any $\epsilon>0$, there exists $\delta>0$ such that, for all $u, v \in \mathbb{B}(\bar{u} ; \delta), \bar{u}^{*} \in \partial f(\bar{u})$, one has

$$
f(v)-f(u) \geqq\left\langle\bar{u}^{*}, v-u\right\rangle-e\|v-u\|, \quad \text { where } \quad e=(\epsilon, \ldots, \epsilon) \in \operatorname{int} \mathbb{R}_{+}^{m} .
$$

From now onwards, we always assume that the subset $X \subseteq \mathbb{R}^{n}$ is a nonempty invex set with respect to some $\eta$ unless otherwise specified.

Wang et al. [28] presented the notions of approximate invexity of higher order with respect to the two kernel functions. Motivated by the work of Wang et al. [28] we are introducing the definition of approximate invexity (of order one) with respect to the single kernel function.

Definition 2.3. A locally Lipschitz vector function $f: X \rightarrow \mathbb{R}^{m}$ is said to be approximate invex at $\bar{u} \in X$, with respect to the kernel function $\eta: X \times X \rightarrow \mathbb{R}^{n}$ if for any $\epsilon>0$, there exists $\delta>0$, for all $u \in X, \bar{u}^{*} \in \partial f(\bar{u})$, one has

$$
f(u)-f(\bar{u}) \geqq\left\langle\bar{u}^{*}, \eta(u, \bar{u})\right\rangle-e\|\eta(u, \bar{u})\|, \quad \text { where } \quad e=(\epsilon, \ldots, \epsilon) \in \operatorname{int} \mathbb{R}_{+}^{m} .
$$

Example 2.4. Let $X=\mathbb{R}$ and $e=\left(\epsilon_{1}, \epsilon_{2}\right)^{T}>0$. Consider the following functions: $f: X \rightarrow \mathbb{R}^{2}, \eta: X \times X \rightarrow \mathbb{R}$ defined by

$$
f(u)=(u, \max \{-u, 0, u-1\})^{T}, \quad \eta(u, \bar{u})=u-\bar{u} .
$$

Take $\bar{u}=-1$, then we have $\bar{u}^{*}=(1,-1)^{T}$. It follows that,

$$
f(u)-f(\bar{u})=(u, \max \{-u, 0, u-1\})^{T}-(-1,1)^{T}=(u+1, \max \{-u, 0, u-1\}-1)^{T},
$$

and

$$
\begin{aligned}
\left\langle\bar{u}^{*}, \eta(u, \bar{u})\right\rangle-e\|\eta(u, \bar{u})\| & =(1,-1)^{T}(u+1)-\left(\epsilon_{1}, \epsilon_{2}\right)^{T}\|u+1\| \\
& =(u+1,-(u+1))^{T}-\left(\epsilon_{1}, \epsilon_{2}\right)^{T}\|u+1\| .
\end{aligned}
$$

Obviously,

$$
(u+1, \max \{-u, 0, u-1\}-1)^{T} \geqq(u+1,-(u+1))^{T} .
$$

Since, $e=\left(\epsilon_{1}, \epsilon_{2}\right)^{T}>0$, we can arrive at

$$
(u+1, \max \{-u, 0, u-1\}-1)^{T} \geqq(u+1,-(u+1))^{T}-\left(\epsilon_{1}, \epsilon_{2}\right)^{T}\|u+1\| .
$$

That is

$$
f(u)-f(\bar{u}) \geqq\left\langle\bar{u}^{*}, \eta(u, \bar{u})\right\rangle-e\|\eta(u, \bar{u})\| .
$$

So, we have verified that $f$ is approximately invex at $\bar{u}=-1$ with respect to $\eta$.

Now, we provide an example which is approximate invex but not approximate convex.

Example 2.5. Let $f: \mathbb{R} \rightarrow \mathbb{R}$ is given by $f(u)=\left\{\begin{array}{ll}0, & u \leqslant 0 \\ u, & u>0\end{array}\right.$ then at $\bar{u}=0$, we have $\bar{u}^{*}=[0,1]$. Now, suppose $f$ is approximate convex at $\bar{u}=0$ then for all $u \in \mathbb{R}$ and $\epsilon>0$, we have

$$
f(u)-f(\bar{u})-\left\langle\bar{u}^{*}, u-\bar{u}\right\rangle \geqslant \epsilon\|u-\bar{u}\| .
$$

If we take $u>0$, and $\epsilon=2$, then the above assumption contradicts. That is function is not approximate convex at $\bar{u}=0$.

Now, if we take, $\bar{u}=0$ and $\eta(u, \bar{u})=u \sin \bar{u}$, then following inequality holds

$$
f(u)-f(\bar{u})-\left\langle\bar{u}^{*}, \eta(u, \bar{u})\right\rangle \geqslant \epsilon\|\eta(u, \bar{u})\| .
$$

That is $f$ with respect to the kernel function $\eta$ satisfies (2.1). So function $f$ is approximate invex at $\bar{u}=0$, with respect to the given kernel function $\eta$. 
Interested reader is referred to [28] for more details on approximated invex functions.

In the next section, we will define approximate Minty type and Stampacchia type vector variational inequalities.

\section{Approximate Vector VARiational inequalities}

In this section, we consider the nonsmooth vector optimization problem as follows:

$$
\text { (VOP) } \min f(u) \text { subject to } u \in X,
$$

where $f: X \rightarrow \mathbb{R}^{m}$ is locally Lipschitz on $X$ and approximately invex at $\bar{u} \in X$. A vector $\bar{u} \in X$ is said to be an efficient solution of the VOP, iff there is no vector $u \in X$ such that

$$
f(u) \leqslant f(\bar{u}) .
$$

When (3.1) is true for all $u$ in a small neighborhood of $\bar{u}$, we say that $\bar{u}$ is a local efficient solution of the VOP. The following concepts of efficient solutions (AES) is a variant of the concept of approximate efficient solution introduced in [18].

Definition 3.1. (a) A vector $\bar{u} \in X$ is said to be an approximate efficient solution of type one with respect to the kernel function $\eta$ of the VOP, denoted by (AES) $)_{1}$, iff for any $\epsilon>0$ sufficiently small, there does not exist $\delta>0$ such that, for all $u \in \mathbb{B}(\bar{u}, \delta) \backslash\{\bar{u}\}$, one has

$$
f(u)-f(\bar{u}) \leqslant e\|\eta(u, \bar{u})\| .
$$

(b) A vector $\bar{u} \in X$ is said to be an efficient solution of type two with respect to the kernel function of the VOP, denoted by $(\mathrm{AES})_{2}$, iff for any $\epsilon>0$ sufficiently small, there exists $\delta>0$ such that, for all $u \in \mathbb{B}(\bar{u}, \delta)$, one has

$$
f(u)-f(\bar{u}) \nless e\|\eta(u, \bar{u})\| .
$$

(c) A vector $\bar{u} \in X$ is said to be an efficient solution of type three with respect to the kernel function of the VOP, denoted by $(\mathrm{AES})_{3}$, iff for any $\epsilon>0$, there exists $\delta>0$ such that, for all $u \in \mathbb{B}(\bar{u}, \delta)$, one has

$$
f(u)-f(\bar{u}) \nless-e\|\eta(u, \bar{u})\| .
$$

Remark 3.2. An equivalent definition of the above solutions can be given as follows.

(a) A vector $\bar{u} \in X$ is an (AES) $)_{1}$, iff there is a sequence of vectors $\left\{u^{y}\right\}_{y \in \mathbb{N}}$ converging to $\bar{u}$ such that $f_{-e}\left(u^{y}\right) \not \nless$ $f_{-e}(\bar{u})$ for every $y \in \mathbb{N}$, where $f_{-e}(u)=f(u)-e\|\eta(u, \bar{u})\|$.

(b) A vector $\bar{u} \in X$ is an $(\mathrm{AES})_{2}$, iff it is a local efficient solution of the problem of minimizing the function $f_{-e}(u)$ over $X$.

(c) A vector $\bar{u} \in X$ is an (AES) $)_{3}$, iff it is a local efficient solution of the problem of minimizing the function $f_{e}(u)=f(u)+e\|\eta(u, \bar{u})\|$ over $X$.

An $(\mathrm{AES})_{2}$ is a strengthened notion of local efficiency while an $(\mathrm{AES})_{3}$ is a weakened notion of local efficiency. Moreover, an (AES) ${ }_{1}$ can be regarded as a quasi-efficient solution of the VOP. It is clear that an $(\mathrm{AES})_{2}$ of the $\mathrm{VOP}$ is also an $(\mathrm{AES})_{1}$ and an $(\mathrm{AES})_{3}$ of the VOP.

Example 3.3. Consider the multiobjective optimization problem (MOP) as follows:

$$
\min f(u):=\left(f_{1}(u), f_{2}(u)\right) \text { s.t. } u \in \mathbb{R},
$$


where $f_{1}(u):=\sin |u|$ and $f_{2}(u):=|u|-u^{4}$ let $\bar{u}:=0$ and $\eta(u, \bar{u})=u$. For any $\epsilon>0$, one has

$$
\begin{aligned}
& f_{1}(u)-f_{1}(\bar{u})+\epsilon\|\eta(u, \bar{u})\| \geqslant \sin |u| \geqslant 0, \quad \forall u \in[-\pi, \pi], \\
& f_{2}(u)-f_{2}(\bar{u})+\epsilon\|\eta(u, \bar{u})\| \geqslant|u|-u^{4} \geqslant 0, \quad \forall u \in[-1,1] .
\end{aligned}
$$

Choose $0<\delta<1$, one has

$$
f(u)-f(\bar{u})+e\|\eta(u, \bar{u})\| \notin-\mathbb{R}_{+}^{2} \backslash\{0\}, \quad \forall u \in \mathbb{B}(0, \delta) .
$$

Hence, $\bar{u}:=0$ is $(\mathrm{AES})_{3}$ of the MOP.

Example 3.4. Consider $f_{1}(u)=|u|-u^{2}$ and $f_{2}(u)=u^{3}-u^{2}$, let $\bar{u}:=0$ and $\eta(u, \bar{u})=u$. For given $0<\epsilon<1$, one has

$$
f_{1}(u)-f_{1}(\bar{u})-\epsilon\|\eta(u, \bar{u})\|=|u|-u^{2}-\epsilon|u| \geqslant 0, \quad \forall u \in[-(1-\epsilon),(1-\epsilon)] .
$$

Also

$$
f_{2}(u)-f_{2}(\bar{u})-\epsilon\|\eta(u, \bar{u})\|=u^{3}-u^{2}-\epsilon|u| \geqslant 0, \quad \forall u \geqslant \frac{1+\sqrt{1+4 \epsilon}}{2} .
$$

Choose $0<\delta<(1-\epsilon)$. Then

$$
f(u)-f(\bar{u})-e\|\eta(u, \bar{u})\| \notin-\mathbb{R}_{+}^{2} \backslash\{0\}, \quad \forall u \in \mathbb{B}(0, \delta) .
$$

Hence, $\bar{u}:=0$ is $(\mathrm{AES})_{2}$ of the MOP. Similarly, one can see that $\bar{u}:=1$ is also an (AES) $)_{2}$ of the MOP.

Example 3.5. In the MOP of Example 3.3, let $\bar{u}:=0$. Proceeding as in Example 3.4, it can be shown that, for given $0<\epsilon<1$ and for any $\delta>0$, there exists $u \in \mathbb{B}(\bar{u}, \delta) \backslash\{\bar{u}\}$ such that

$$
f(u)-f(\bar{u})-e\|\eta(u, \bar{u})\| \notin-\mathbb{R}_{+}^{2} \backslash\{0\},
$$

which implies that $\bar{u}:=0$ is an $(\mathrm{AES})_{1}$ of the MOP.

\subsection{Approximate Minty type vector variational inequality}

The following vector variational inequalities of Minty type will be used in the sequel to characterize an approximate efficient solution of the VOP.

$(\mathbf{A M V})_{\mathbf{1}}$ To find $\bar{u} \in X$ such that, for any $\epsilon>0$ sufficiently small, there does not exist $\delta>0$ such that, for all $u \in \mathbb{B}(\bar{u}, \delta) \backslash\{\bar{u}\}$ and $u^{*} \in \partial f(u)$, one has

$$
\left\langle u^{*}, \eta(u, \bar{u})\right\rangle \leqslant e\|\eta(u, \bar{u})\| .
$$

$(\mathbf{A M V})_{\mathbf{2}}$ To find $\bar{u} \in X$ such that, for any $\epsilon>0$ sufficiently small, there exists $\delta>0$ such that, for all $u \in \mathbb{B}(\bar{u}, \delta)$ and $u^{*} \in \partial f(u)$, one has

$$
\left\langle u^{*}, \eta(u, \bar{u})\right\rangle \nless e\|\eta(u, \bar{u})\| .
$$

$(\mathbf{A M V})_{3}$ To find $\bar{u} \in X$ such that, for any $\epsilon>0$, there exists $\delta>0$ such that, for all $u \in \mathbb{B}(\bar{u}, \delta)$ and $u^{*} \in \partial f(u)$, one has

$$
\left\langle u^{*}, \eta(u, \bar{u})\right\rangle \nless-e\|\eta(u, \bar{u})\| .
$$

Special cases: Following are some special cases in which we have related our present vector variational inequalities with the variational inequalities existed in the literature. 
- Assume that $\eta(u, \bar{u})=u-\bar{u}$ then $(\mathrm{AMV})_{1}$ reduces to approximate weak vector variational inequalities [18]: for finding $\bar{u} \in X$, such that for any, $\epsilon>0$ there does not exist $\delta>0$ such that, for all $i=1,2, \ldots, m, \bar{u}_{i}{ }^{*} \in$ $\partial^{F} f_{i}(\bar{u})$ and $u \in B(\bar{u}, \delta)$, one has

$$
\left\langle\bar{u}_{i}^{*}, u-\bar{u}\right\rangle<\epsilon\|u-\bar{u}\|, \quad u \neq \bar{u}
$$

where $\partial^{F} f(\bar{u})$ stands for Frechet subdifferentials [2] of the function $f$ at point $\bar{u}$.

- If in $(\mathrm{AMV})_{1}$, we put $\eta(u, \bar{u})=u-\bar{u}$ and $\epsilon=0$ then (AMV) $)_{1}$ reduces to vector variational inequalities [20]: find a point $\bar{u} \in X$ such that there exist no $u \in X$ such that

$$
\left\langle\bar{u}^{*}, u-\bar{u}\right\rangle \leqslant 0 ; \quad \forall \bar{u}^{*} \in \partial f(\bar{u}),
$$

where $\partial f(\bar{u})$ stands for Clarke subdifferentials of the function $f$ at point $\bar{u}$.

Remark 3.6. It is also clear that a solution of the $(\mathrm{AMV})_{2}$ is also a solution of the $(\mathrm{AMV})_{1}$ and $(\mathrm{AMV})_{3}$, but the converse does not always hold.

The following theorem gives an approximate version of the Minty variational principle for an (AES) ${ }_{1}$ of the VOP in terms of the $(\mathrm{AMV})_{1}$ and $(\mathrm{AMV})_{2}$.

Theorem 3.7. Let $f$ be locally Lipschitz on $X$ and approximately invex at $\bar{u} \in X$ with respect to the kernel function $\eta$. Then, the following statements hold:

(a) If $\bar{u}$ is an $(\mathrm{AES})_{1}$ of the $\mathrm{VOP}$, then it is also a solution of the (AMV) 1 with respect to the common kernel function $\eta$;

(b) If $\bar{u}$ is a solution of the (AMV $)_{2}$, then it is also an (AES $)_{2}$ with respect to the kernel function $\eta$ of the VOP;

(c) If $\bar{u}$ is an $(\mathrm{AES})_{3}$ of the $\mathrm{VOP}$, then it is also a solution of the (AMV) $)_{3}$ with respect to the common kernel function $\eta$.

Proof. (a) Suppose to the contrary that $\bar{u}$ is not a solution of the (AMV) $)_{1}$. Then, for some $\bar{\epsilon}>0$ sufficiently small, there exists $\bar{\delta}>0$ such that, for all $u \in \mathbb{B}(\bar{u}, \bar{\delta})$ and $u^{*} \in \partial f(u)$, one has

$$
\left\langle u^{*}, \eta(u, \bar{u})\right\rangle \leqslant \frac{\bar{e}}{2}\|\eta(u, \bar{u})\|
$$

where $\bar{e}:=(\bar{\epsilon}, \ldots, \bar{\epsilon}) \in \operatorname{int} \mathbb{R}_{+}^{m}$. Since $f$ is locally Lipschitz on $X$ and approximately invex at $\bar{u}$, with respect to the common kernel function $\eta$, it follows that, for any $\epsilon>0$, there exists $\hat{\delta}>0$ such that, for all $u \in \mathbb{B}(\bar{u}, \hat{\delta})$ and $u^{*} \in \partial f(\bar{u})$, one has

$$
f(\bar{u})-f(u) \geqq\left\langle u^{*}, \eta(\bar{u}, u)\right\rangle-\frac{e}{2}\|\eta(\bar{u}, u)\| .
$$

By setting $\tilde{\delta}:=\min \{\bar{\delta}, \hat{\delta}\}$, it follows that, for some $\bar{\epsilon}>0$ sufficiently small, there exists $\tilde{\delta}>0$ such that, for all $u \in \mathbb{B}(\bar{u}, \tilde{\delta})$ one has

$$
f(u)-f(\bar{u}) \leqslant \bar{e}\|\eta(u, \bar{u})\|,
$$

a contradiction to the fact that $\bar{u}$ is an $(\mathrm{AES})_{1}$ of the VOP. This completes the proof.

(b) Suppose to the contrary that $\bar{u} \in X$ is not an (AES) $)_{2}$ of the VOP. Then, for some $\bar{\epsilon}>0$ sufficiently small and for every $\delta>0$ one finds some $u \in \mathbb{B}(\bar{u}, \delta) \backslash\{\bar{u}\}$ such that

$$
f(u)-f(\bar{u}) \leqslant \frac{\bar{e}}{2}\|\eta(u, \bar{u})\|
$$

For this $\bar{\epsilon}$, we choose $\bar{\delta}>0$, so that the relation given in $(\mathrm{AMV})_{2}$ holds. Then, with this $\bar{\delta}$, let $u \in \mathbb{B}(\bar{u}, \bar{\delta})$ be such that

$$
f\left(u_{0}\right)-f(\bar{u}) \leqslant \frac{\bar{e}}{2}\|\eta(u, \bar{u})\| .
$$


Applying the mean value theorem, there exist $u_{0}^{*} \in \operatorname{co}\left(\partial f\left(\left[\bar{u}, u_{0}\right]\right)\right)$ such that

$$
f\left(u_{0}\right)-f(\bar{u})=\left\langle u_{0}^{*}, \eta_{0}(u, \bar{u})\right\rangle .
$$

Accordingly there exist $u_{1}, \ldots, u_{k}$ from the open segment $\left(\bar{u}, u_{0}\right), u_{1}^{*} \in \partial f\left(u_{1}\right), \ldots, u_{k}^{*} \in \partial f\left(u_{k}\right), 0<\beta_{1}<$ $, \ldots,<\beta_{k}<1, \alpha_{1}, \ldots, \alpha_{k}>0$ such that

$$
\begin{aligned}
\alpha_{1}+\alpha_{1}, \ldots, \alpha_{k} & =1 ; \quad u_{0}^{*}=\alpha_{1} u_{1}^{*}+\alpha_{2} u_{2}^{*}+\ldots+\alpha_{k} u_{k}^{*}=u_{1}^{*}+\sum_{i=2}^{k} \alpha_{i}\left(u_{i}^{*}-u_{1}^{*}\right) \\
\eta_{i}(u, \bar{u}) & =\beta_{i} \eta_{0}(u, \bar{u}), \quad i=1, \ldots, k
\end{aligned}
$$

By approximate invexity of $f$ at $\bar{u}$, with respect to the kernel function $\eta$, it follows that

$$
\left\langle u_{i}^{*}-u_{1}^{*}, \eta_{i}\left(u, u_{1}\right)\right\rangle \geqq-\frac{e}{2}\left\|\eta_{i}\left(u, u_{1}\right)\right\|, \quad \forall i=1,2, \ldots, k
$$

from (3.3) to (3.5), it follows that

$$
\begin{aligned}
\left\langle u_{0}^{*}, \eta_{0}(u, \bar{u})\right\rangle & =\left\langle u_{1}^{*}, \eta_{0}(u, \bar{u})\right\rangle+\sum_{i=2}^{k} \alpha_{i}\left\langle u_{i}^{*}-u_{1}^{*}, \eta_{0}(u, \bar{u})\right\rangle \\
& =\frac{1}{\beta_{1}}\left\langle u_{1}^{*}, \eta_{1}(u, \bar{u})\right\rangle+\sum_{i=2}^{k} \frac{\alpha_{i}}{\beta_{i}-\beta_{1}}\left\langle u_{i}^{*}-u_{1}^{*}, \eta_{i}\left(u, u_{1}\right)\right\rangle \leqslant \frac{\bar{e}}{2}\left\|\eta_{0}(u, \bar{u})\right\|
\end{aligned}
$$

from (3.6) it follows that

$$
\frac{1}{\beta_{1}}\left\langle u_{1}^{*}, \eta_{1}(u, \bar{u})\right\rangle \leqslant \frac{\bar{e}}{2}\left\|\eta_{0}(u, \bar{u})\right\|+\frac{\bar{e}}{2} \sum_{i=2}^{k} \frac{\alpha_{i}}{\beta_{i}-\beta_{1}}\left\|\eta_{i}\left(u, u_{1}\right)\right\| .
$$

Consequently, from (3.5), it follows that

$$
\left\langle u_{1}^{*}, \eta_{1}(u, \bar{u})\right\rangle \leqslant \frac{\bar{e}}{2}\left(1+\sum_{1=2}^{k} \alpha_{i}\right)\left\|\eta_{1}(u, \bar{u})\right\| \leqq \bar{e}\left\|\eta_{1}(u, \bar{u})\right\|,
$$

with $u_{1} \in\left[\bar{u}, u_{0}\right] \subset \mathbb{B}(\bar{u}, \bar{\delta})$ and $u_{1}^{*} \in \partial f\left(u_{1}\right)$. This contradicts the fact that $\bar{u}$ is a solution of the $(\mathrm{AMV})_{2}$.

(c) We proceed as for (a). Suppose that $\bar{u}$ is an (AES) $)_{3}$ of the VOP. Then, for any $\epsilon>0$, there exists $\bar{\delta}>0$ such that, for all $u \in \mathbb{B}(\bar{u}, \bar{\delta})$ one has

$$
f(u)-f(\bar{u}) \nless-\frac{e}{2}\|\eta(u, \bar{u})\| .
$$

Since $f$ is locally Lipschitz on $X$ and approximately invex at $\bar{u}$, with respect to the kernel function $\eta$, i.e. for any $\epsilon>0$, there exists $\tilde{\delta}>0$ such that, for all $u \in \mathbb{B}(\bar{u}, \tilde{\delta})$ and $u^{*} \in \partial f(u)$, one has

$$
f(\bar{u})-f(u) \geqq\left\langle u^{*}, \eta(\bar{u}, u)\right\rangle-\frac{e}{2}\|\eta(\bar{u}, u)\| .
$$

Setting $\hat{\delta}:=\min \{\bar{\delta}, \tilde{\delta}\}$, it follows that, for any $\epsilon>0$, there exists $\hat{\delta}>0$ such that, for all $u \in \mathbb{B}(\bar{u}, \hat{\delta})$ and $u^{*} \in \partial f(u)$, one has

$$
\left\langle u^{*}, \eta(u, \bar{u})\right\rangle \nless-e\|\eta(u, \bar{u})\| .
$$

Hence, $\bar{u}$ is a solution of the $(\mathrm{AMV})_{3}$. This completes the proof. 


\subsection{Approximate Stampacchia type vector variational inequality}

Now, we consider the approximate Stampacchia type vector variational inequalities as follows:

$(\mathbf{A S V})_{1}$ To find $\bar{u} \in X$ such that, for any $\epsilon>0$ sufficiently small, there are some $u \in X \backslash\{\bar{u}\}$ and $\bar{u}^{*} \in \partial f(\bar{u})$ satisfying

$$
\left\langle\bar{u}^{*}, \eta(u, \bar{u})\right\rangle \nless k\|\eta(u, \bar{u})\| .
$$

$(\mathbf{A S V})_{2}$ To find $\bar{u} \in X$ such that for any $\epsilon>0$ sufficiently small, for every $u \in X$ and $\bar{u}^{*} \in \partial f(\bar{u})$, one has

$$
\left\langle\bar{u}^{*}, \eta(u, \bar{u})\right\rangle \nless e\|\eta(u, \bar{u})\| .
$$

$(\mathbf{A S V})_{3}$ To find $\bar{u} \in X$ such that, for any $\epsilon>0$, for all $u \in \mathbb{B}(\bar{u}, \delta)$ and $\bar{u}^{*} \in \partial f(\bar{u})$, one has

$$
\left\langle\bar{u}^{*}, \eta(u, \bar{u})\right\rangle \nless-e\|\eta(u, \bar{u})\| .
$$

Remark 3.8. A solution of the $(\mathrm{ASV})_{2}$ is also a solution of the $(\mathrm{ASV})_{1}$ and the $(\mathrm{ASV})_{3}$.

The following theorem gives the relationship between solutions of the variational inequality problems of Stampacchia type and efficient solutions of the VOP.

Theorem 3.9. Let $f$ be locally Lipschitz on $X$ and approximately invex at $\bar{u} \in X$. If $\bar{u}$ is a solution of the $(\mathrm{ASV})_{1}$ (respectively $(\mathrm{ASV})_{2}$ and $\left.(\mathrm{ASV})_{3}\right)$, then $\bar{u}$ is also an $(\mathrm{AES})_{1}$ (respectively $(\mathrm{AES})_{2}$ and $\left.(\mathrm{AES})_{3}\right)$ of the VOP.

Proof. Let $\bar{u}$ be a solution of the $(\mathrm{ASV})_{1}$ and suppose to the contrary that it is not an $(\mathrm{AES})_{1}$. There exist $\epsilon>0$ and $\delta>0$ such that

$$
f(u)-f(\bar{u}) \leqslant e\|\eta(u, \bar{u})\|
$$

for all $u \in \mathbb{B}(\bar{u}, \delta), x \neq \bar{u}$. Since $f$ is approximate invex at $\bar{u}$, with respect to the kernel function $\eta$, i.e. there is $\delta^{\prime}<\delta$ such that

$$
\left\langle\bar{u}^{*}, \eta(u, \bar{u})\right\rangle \leqq f(u)-f(\bar{u})
$$

for all $u \in \mathbb{B}\left(\bar{u}, \delta^{\prime}\right)$ and $\bar{u}^{*} \in \partial f(\bar{u})$. Then

$$
\left\langle\bar{u}^{*}, \eta(u, \bar{u})\right\rangle \leqq e\|\eta(u, \bar{u})\|
$$

for all $u \in \mathbb{B}(\bar{u}, \delta), u \neq \bar{u}$, and hence for all $u \in X \backslash\{\bar{u}\}$. This contradicts the fact that $\bar{u}$ solves (ASV) $)_{1}$.

Next we show that if $\bar{u}$ is a solution of $(\mathrm{ASV})_{2}$, then it is a solution of $(\mathrm{AMV})_{2}$, which in its turn is an $(\mathrm{AES})_{2}$ by Theorem 3.7. Indeed, for every $\epsilon>0$, for every $u \in X$ and $\bar{u}^{*} \in \partial f(\bar{u})$, we have

$$
\left\langle\bar{u}^{*}, \eta(u, \bar{u})\right\rangle \nless \frac{e}{2}\|\eta(u, \bar{u})\| .
$$

Since $f$ is approximate invex, for $u$ sufficiently close to $\bar{u}$, we have

$$
\left\langle u^{*}-\bar{u}^{*}, \eta(u, \bar{u})\right\rangle \geqq-\frac{e}{2}\|\eta(u, \bar{u})\| \text { for all } u^{*} \in \partial f(\bar{u}) .
$$

Consequently,

$$
\left\langle u^{*}, \eta(u, \bar{u})\right\rangle \nless e\|\eta(u, \bar{u})\|
$$

for all $u$ in a small neighborhood of $\bar{u}$ and for all $u^{*} \in \partial f(u)$. By this $\bar{u}$ is a solution of $(\mathrm{AMV})_{2}$. Finally, let $\bar{u}$ is a solution of the $(\mathrm{ASV})_{3}$. Then, for any $\epsilon>0$, there exists $\hat{\delta}>0$ such that, for all $u \in \mathbb{B}(\bar{u}, \hat{\delta})$ and $\bar{u}^{*} \in \partial f(\bar{u})$, one has

$$
\left\langle\bar{u}^{*}, \eta(u, \bar{u})\right\rangle \nless-\frac{e}{2}\|\eta(u, \bar{u})\| .
$$


Since $f$ is locally Lipschitz on $X$ and approximately invex at $\bar{u}$, with respect to the common kernel function $\eta$, i.e. for any $\epsilon>0$, there exists $\bar{\delta}>0$ such that, for all $u \in \mathbb{B}(\bar{u}, \bar{\delta})$ and $\bar{u}^{*} \in \partial f(\bar{u})$, one has

$$
f(u)-f(\bar{u}) \geqq\left\langle\bar{u}^{*}, \eta(u, \bar{u})\right\rangle-\frac{e}{2}\|\eta(u, \bar{u})\| .
$$

Setting $\tilde{\delta}:=\{\hat{\delta}, \bar{\delta}\}$, it follows that, for any $\epsilon>0$, there exists $\tilde{\delta}>0$ such that, for all $u \in \mathbb{B}(\bar{u}, \tilde{\delta})$ one has

$$
f(u)-f(\bar{u}) \nless-e\|\eta(u, \bar{u})\| .
$$

Hence, $\bar{u}$ is an $(\mathrm{AES})_{3}$ of the VOP. This completes the proof.

Example 3.10. Consider the VOP as follows:

$$
(\mathrm{VOP}) \min f(u):=\left(f_{1}(u), f_{2}(u)\right) \text { s.t. } u \in \mathbb{R},
$$

where $f_{1}(u):=2 u^{3}-3 u^{2}$ and $f_{2}(u):=u^{3}-2 u$. For $\bar{u}:=0$, one has

$$
f_{1}(u)-f_{1}(\bar{u})=2 u^{3}-3 u^{2}<0 \quad \forall u \in(0,1.5),
$$

and

$$
f_{2}(u)-f_{2}(\bar{u})=u^{3}-2 u<0 \quad \forall u \in(0,1.4) .
$$

Hence, $\bar{u}:=0$ is neither a weak efficient solution of the VOP nor a local weak efficient solution of the VOP.

Now, it is easy to see that for any $\epsilon>0$, there exists $\delta>0$ such that, for all $u \in \mathbb{B}(\bar{u}, \delta)$, one has

$$
\left(f_{1}(u)-f_{1}(\bar{u})+\epsilon\|\eta(u, \bar{u})\|, f_{2}(u)-f_{2}(\bar{u})+\epsilon\|\eta(u, \bar{u})\|\right) \notin-\mathbb{R}_{+}^{2} \backslash\{0\} .
$$

Hence, $\bar{u}:=0$ is $(\mathrm{AES})_{3}$ of the VOP.

Acknowledgements. The author is thankful to the anonymous referees for their valuable comments and suggestions which helped to improve the presentation of the paper.

\section{REFERENCES}

[1] T. Amahroq, J.-P. Penot and A. Syam, On the subdifferentiability of difference of two functions and local minimization. Set Valued Anal. 16 (2008) 413-427.

[2] J.M. Borwein and Q.J. Zhu, Techniques of Variational Analysis. Springer-Verlag (2005).

[3] R.I. Bot and D.-M. Nechita, On the Dini-Hadamard subdifferential of the difference of two functions. J. Global Optim. 50 (2011) 485-502.

[4] Z. Chen, Asymptotic analysis for proximal-type methods in vector variational inequality problems. Oper. Res. Lett. 43 (2015) 226-230.

[5] B.D. Craven, Invex function and constrained local minima. Bull. Aust. Math. Soc. 24 (1981) 357-366.

[6] A. Daniilidis and P. Georgiev, Approximate convexity and submonotonicity. J. Math. Anal. App. 291 (2004) $292-301$.

[7] F. Facchinei and J.-S. Pang, Finite-Dimensional Variational Inequalities and Complementarity Problems. Springer, New York (2003).

[8] M.C. Ferris and J.S. Pang, Engineering and economic applications of complementarity problems. SIAM Rev. 39 (1997) 669-713.

[9] F.F. Guo, L.W. Zhang and Y.H. Ren, Error bounds for affine variational inequalities with second-order cone constraints. Oper. Res. Lett. 45 (2017) 456-460.

[10] A. Gupta, A. Mehra and D. Bhatia, Approximate convexity in vector optimization. Bull. Aust. Math. Soc. 74 (2006) $207-218$.

[11] C. Gutiérrez, B. Jiménez, V. Novo and G. Ruiz-Garzón, Vector critical points and efficiency in vector optimization with Lipschitz functions. Optim. Lett. 10 (2016) 47-62.

[12] M.A. Hanson, On sufficiency of the Kuhn-Tucker conditions. J. Math. Anal. App. 80 (1981) 545-550.

[13] V.I. Ivanov, Second-order invex functions in nonlinear programming. Optimization 61 (2012) 489-503.

[14] Y. Jiang, X. Cai and D. Han, Solving policy design problems: alternating direction method of multipliers-based methods for structured inverse variational inequalities. Eur. J. Oper. Res. 280 (2020) 417-427.

[15] A. Jofre, D.T. Luc and M. Thera, $\epsilon$-subdifferential and $\epsilon$-monotonicity. Nonlinear Anal. 33 (1998) 71-90. 
[16] P. Loridan, $\epsilon$-in vector minimization problems. J. Optim. Theory App. 43 (1984) 265-276.

[17] S.K. Mishra and G. Giorgi, Invexity and Optimization. In: Vol. 88 of Nonconvex Optimization and Its Applications. Springer, Berlin (2008).

[18] S.K. Mishra and V. Laha, On approximately star-shaped functions and approximate vector variational inequalities. J. Optim. Theory App. 156 (2012) 278-293.

[19] S.K. Mishra and V. Laha, On minty variational principle for nonsmooth vector optimization problems with approximate convexity. Optim. Lett. 10 (2016) 577-589.

[20] S.K. Mishra and B.B. Upadhyay, Some relations between vector variational inequality problems and nonsmooth vector optimization problems using quasi efficiency. Positivity 17 (2013) 1071-1083.

[21] S. Mititelu, Invex sets. Stud. Cerc. Mat. 46 (1994) 529-532.

[22] H.V. Ngai and J.-P. Penot, Approximately convex functions and approximately monotonic operators. Nonlinear Anal. 66 (2007) 547-564.

[23] H.V. Ngai and J.-P. Penot, Semismoothness and directional subconvexity of functions. Pac. J. Optim. 3 (2007) $323-344$.

[24] H.V. Ngai, D.T. Luc and M. Thera, Approximate convex functions. J. Nonlinear Convex Anal. 1 (2000) $155-176$.

[25] R. Osuna-Gómez, A. Rufián-Lizana and P. Ruiz-Canales, Invex functions and generalized convexity in multiobjective programming. J. Optim. Theory App. 98 (1998) 651-661.

[26] J.-P. Penot, The directional subdifferential of the difference of two convex functions. J. Global Optim. 49 (2011) 505-519.

[27] G.J. Tang, M. Zhu and H.W. Liu, A new extragradient-type method for mixed variational inequalities. Oper. Res. Lett. 43 (2015) 567-572.

[28] Z. Wang, R. Li and G. Yu, Vector critical points and generalized quasi-efficient solutions in nonsmooth multi-objective programming. J. Inequal. App. (2017) 1-12.

[29] D.J. White, Epsilon efficiency. J. Optim. Theory App. 49 (1986) 319-337. 